\title{
AVALIAÇÃO DA QUALIDADE DE VIDA NO TRABALHO DE PROFISSIONAIS EM UMA UNIDADE SAÚDE DA FAMÍLIA
}

\section{EVALUATION OF QUALITY OF WORKING LIFE OF PROFESSIONALS FROM FAMILY HEALTH UNIT}

\author{
Felipe Muniz de Andrade ${ }^{\text {** }}$; Gabriella Barreto Soares II
}

\begin{abstract}
Resumo. O debate sobre Qualidade de Vida no Trabalho (QVT) na Estratégia Saúde da Família (ESF) é importante, pois envolve dimensões intelectuais e desenvolvimento de diferentes técnicas e competências para o manejo da saúde individual, da família e da comunidade, cursando assim com maior carga física e mental para os profissionais de saúde. Com isso, o objetivo desse estudo é avaliar a QVT de trabalhadores de uma Unidade de Saúde da Família (USF) de João Pessoa, Paraíba. Trata-se de estudo quantitativo, descritivo e transversal, com aplicação de formulário sociodemográfico e questionário QWLQ-bref na USF Integrando Vidas, Distrito Sanitário II, em fevereiro de 2021. De 60 profissionais, obteve-se índice 64,86, considerado satisfatório pela escala de classificação do QWLQ-bref. A única variável que diferiu da classificação total foi a categoria profissional de técnico de enfermagem ou laboratório, com índice 47,11, considerado neutro (nem boa, nem ruim). O nível satisfatório de QVT pode ser relacionado às características da USF, como possuir equipes de Saúde da Família completas, com atuação de programas de residência multiprofissional e médica. No entanto, existem fatores que poderiam ser melhorados como o índice do domínio profissional menor que os demais, bem como a classificação inferior da categoria de técnicos, destacando a falta de insumos, problemas de infraestrutura, falta de formação continuada e reuniões de equipe. A QVT dos trabalhadores de saúde da USF Integrando Vidas foi considerada satisfatória, com o domínio pessoal com maior escore e o profissional com o menor.
\end{abstract}

PALAVRAS-CHAVE: Qualidade de Vida. Pessoal de Saúde. Estratégia Saúde da Família.

Abstract. The debate on Quality of Life at Work (QWL) in the Family Health Strategy (ESF) is important, as it involves intellectual dimensions and the development of different techniques and skills for the management of individual, family and community health, thus progressing with greater physical and mental burden for health professionals. Thus, the aim of this study is to assess the QWL of workers at a Family Health Unit (USF) in João Pessoa, Paraíba. This is a quantitative, descriptive and cross-sectional study, with the application of a sociodemographic form and QWLQ-bref questionnaire at the USF Integrando Vidas, Sanitary District II, in February 2021. From 60 professionals, an index of 64.86 was obtained, considered satisfactory by QWLQ-bref rating scale. The only variable that differed from the total classification was the professional category of nursing technician or laboratory, with an index of 47.11 , considered neutral (neither good nor bad). The satisfactory level of QWL can be related to the characteristics of the USF, such as having complete Family Health teams, with multidisciplinary and medical residency programs. However, there are factors that could be improved, such as the lower professional domain index than the others, as well as the lower classification of the technicians category, highlighting the lack of inputs, infrastructure problems, lack of continuing education and team meetings. The QWL of health workers at the USF Integrando Vidas was considered satisfactory, with the personal domain with the highest score and the professional with the lowest.

KEYWORDS: Quality of Life. Health Personnel. Family Health Strategy. 


\section{INTRODUÇÃO}

O ser humano tem no trabalho grande parte de sua dedicação, investindo tempo, conhecimento e esforço. A competitividade pelo mercado de trabalho e a demanda cada vez maior por resultados ideais faz o emprego ter um peso importante na vida do indivíduo, influenciando no seu bem-estar. ${ }^{1}$ A satisfação nessa dimensão se relaciona a aspectos laborais, tanto quanto pessoais do profissional, de modo que é imprescindível que esses trabalhadores possam abordar problemas vivenciados na rotina do trabalho, para que se oferte uma assistência integral à sua saúde e se obtenha suporte para solucionar os entraves vivenciados. ${ }^{2}$

Houve uma tendência à humanização do trabalho, após a Revolução Industrial, de forma que a classe operária passou a solicitar melhores jornadas de trabalho, com remunerações mais adequadas, tendo ganhado importância o debate sobre a saúde e o bem-estar do trabalhador, não se observando, no entanto, uma melhora significativa na Qualidade de Vida no Trabalho (QVT). ${ }^{3}$ É visível que a QVT é influenciada por diferentes variáveis, como satisfação com a remuneração, relações interpessoais no ambiente de trabalho e reconhecimento pela função exercida e pelos objetivos atingidos, além de fatores externos como relações familiares e sociais e estado psicológico e físico, influenciando a motivação e, consequentemente, a produtividade. ${ }^{1}$

Nessa conjuntura, também se destaca a micropolítica relacionada com o papel protagonista dos profissionais de saúde e usuários e suas relações, guiados por esforços variados que regem planejamentos em saúde, principalmente na Atenção Básica (AB) no Sistema Único de Saúde (SUS). Vale ressaltar que o âmbito da micropolítica é diversificado devido às identidades existentes e aos seus projetos, e nele se ditam as tecnologias orientadoras da promoção dos cuidados em saúde. ${ }^{4}$

Com esse escopo, a QVT é definida como "o conjunto de ações desenvolvidas pelas empresas na implantação de melhorias gerenciais, estruturais e tecnológicas, na busca da satisfação e do bem-estar físico, psicológico, social e profissional". 5 Para a prestação de uma adequada atenção à saúde pública, é necessário haver trabalhadores satisfeitos e com o aproveitamento de uma boa qualidade de vida. ${ }^{6}$ Contudo, o SUS ainda encontra obstáculos a serem superados ${ }^{7}$, até mesmo porque essas políticas tem sofrido ataques e contingenciamento de recursos.

Dentre os desafios do SUS, a busca e a manutenção de uma qualidade de vida do profissional de saúde tomam seu relevante papel. Com a nova estruturação do processo de trabalho, a Estratégia Saúde da Família (ESF) se torna a esperança para aumentar o acesso, e essa proposta suscitou a discussão sobre a precariedade desse processo de trabalho e suas repercussões ao trabalhador da área da saúde. ${ }^{1}$

$\mathrm{Na}$ Atenção Básica, a associação do modelo de assistência tradicional com o modelo com foco na ESF impõe maior dimensionamento intelectual e de diferentes habilidades e competências no manejo da saúde individual, da família e da coletividade. ${ }^{8}$

Nesse contexto, há um aumento da carga física e mental de trabalho. ${ }^{9}$ No entanto, observa-se hoje uma quantidade mais expressiva de estudos referentes à saúde do trabalhador na área hospitalar, com uma valorização ainda desigual entre os níveis de complexidade da 
assistência. ${ }^{5}$ A ESF, por operacionalizar suas práticas com enfoque na família e na comunidade, precisa de profissionais com tais competências e com qualidade de vida. Não sendo assim, não seria praticável a promoção do cuidado e bem estar a que a estratégia se propõe. ${ }^{10}$

Ainda, não pode deixar de ser considerada, no atual momento histórico, a influência da pandemia do novo coronavírus (COVID-19) no processo de trabalho e qualidade de vida de trabalhadores da ESF, uma vez que vem trazendo necessidade de adaptação de modelos de gestão da saúde, de relações micropolíticas e de como planejar o cuidado direcionado necessário neste momento para promoção, prevenção

\section{MATERIAL E MÉTODOS}

O estudo é caracterizado como quantitativo, descritivo e transversal. A amostra foi composta pelos trabalhadores da USF Integrando Vidas compreendendo uma amostra de sessenta profissionais, que aceitaram participar da pesquisa, e não estavam afastados ou licenciados do serviço. Estavam ativos durante a aplicação do instrumento, número esse com correlação significativa com número amostral de estudos prévios ${ }^{7,10,12}$ A USF pertence ao Distrito Sanitário (DS) II, a qual possui vinculação com os programas de residência médica em MFC e residência multiprofissional em Saúde da Família.

Com uma maior discussão sobre o tema, foram levantados questionários diferentes para que se possa medir a qualidade de vida. ${ }^{1}$ Por meio do estudo, que almejou dispor de abreviação do e educação da população. ${ }^{11}$ Além disso, muitos desses trabalhadores foram linha de frente do enfrentamento da pandemia na maioria dos municípios brasileiros, tendo que lidar com questões de falta de insumos, infraestrutura, desvalorização profissional, pressão assistencial, dentre vários outros fatores.

Diante desse contexto, o objetivo deste estudo é avaliar a QVT de trabalhadores de uma USF do município de João Pessoa, Paraíba, com recorte temporal de fevereiro de 2021, mensurando a QVT de acordo com níveis de satisfação em relação aos domínios físico/saúde, psicológico, pessoal e profissional, identificando os fatores sociodemográficos.

QWLQ-78, foi disponibilizado o QWLQ-bref, validado no Brasil, para avaliação da QVT, com resultados de acordo ao questionário original em menor tempo de pesquisa. $\mathrm{O}$ questionário é composto por 20 questões são organizadas em escala Likert de 5 pontos que englobam quatro domínios: físico/ saúde, psicológico, pessoal e profissional ${ }^{3,5}$ (Tabela 1). Os participantes responderam ao instrumento QLWQ-bref, bem como ao questionário sociodemográfico, incluindo dados como sexo, idade, cor ou raça, grau de escolaridade, categoria profissional, tipo de vínculo, conciliação com outro trabalho, carga horária semanal, renda domiciliar e quantidade de pessoas no domicílio, região geográfica que reside e presença de comorbidade, de modo sigiloso, sendo coletado apenas pelo pesquisador, não sendo identificado o participante. 
TABELA 1: Domínios do QWLQ-78.

\begin{tabular}{ll}
\hline 1. Físico/saúde & $\begin{array}{l}\text { Trata de aspectos relacionados à saúde, doenças relacionadas ao trabalho e } \\
\text { hábitos dos trabalhadores }\end{array}$ \\
\hline 2. Psicológico & $\begin{array}{l}\text { Trata de aspectos relacionados à satisfação pessoal, motivação no trabalho e } \\
\text { auto-estima dos trabalhadores }\end{array}$ \\
\hline 3. Pessoal & $\begin{array}{l}\text { Trata de aspectos relacionados à família, às crenças pessoais e religiosas e à } \\
\text { cultura e de que forma influenciam o trabalho }\end{array}$ \\
\hline 4. Profissional & $\begin{array}{l}\text { Trata de aspectos organizacionais que podem influenciar a vida dos } \\
\text { trabalhadores }\end{array}$ \\
\hline \hline
\end{tabular}

Para tabulação, cálculos e análise dos dados foi utilizada análise descritiva dos dados, por meio de frequência simples. E os dados dos domínios do QWLQ-78 bref foi usada a sintaxe feita para o instrumento, sendo que ao pesquisador compete apenas a tabulação dos dados nos locais especificados em ferramenta do próprio questionário com cálculos realizados de forma automatizada no programa Microsoft Excel para Windows. ${ }^{1,3}$ Os resultados são classificados conforme o Tabela 2, construído por Reis Júnior ${ }^{5}$ para o QWLQ-78.

TABELA 2: Classificação proposta para o QWLQ-78

\begin{tabular}{ccccc}
\hline \hline QWLQ - 78 & & & \\
\hline Muito Insatisfatório & Insatisfatório & Neutro & Satisfatório & Muito Satisfatório \\
o a 22,5 & 22,5 a 45 & 45 a 55 & 55 a 77,5 & 77,5 a 100 \\
\hline & & & Fonte: Reis Junior, 2008.
\end{tabular}

Todos os participantes, antes de responderem $o$ instrumento, assinaram o Termo de Consentimento Livre e Esclarecido - TCLE após serem esclarecidos sobre informações acerca do projeto. O protocolo de pesquisa foi aprovado pelo Comitê de Ética em Pesquisa do Centro de Ciências Médicas da UFPB, conforme parecer $\mathrm{n}^{\circ}$ 4.536.343 e CAAE n 40491020.4.0000.8069.

\section{RESULTADOS E DISCUSSÃO}

O índice de qualidade de vida no trabalho, obtido entre os trabalhadores foi de 64,86 , foi considerado como satisfatório pela escala de classificação de QVT do QWLQ-78 e de sua forma abreviada, conforme ilustrado na Figura 1. Em relação aos domínios, todos foram classificados como satisfatório. O domínio pessoal com maior escore e o profissional com o menor.

A percepção da qualidade de vida no trabalho na ESF foi considerada satisfatória pelos trabalhadores da unidade, o que está de acordo com o relatado por Gomes. ${ }^{10}$ A impressão captada da qualidade de vida dos trabalhadores de USFs foi classificada como boa no domínio geral. ${ }^{10}$ Segundo Bracarense $^{13}$, os entendimentos atribuídos à qualidade de vida pelos trabalhadores da ESF compreendem tanto os aspectos subjetivos, do sentir-se bem no ambiente 
laboral e gostar da profissão, como as questões das condições e relacionamentos oriundos da atividade prestada. O equilíbrio desses fatores pode direcionar uma percepção mais positiva ou negativa do bemestar no âmbito laborativo.

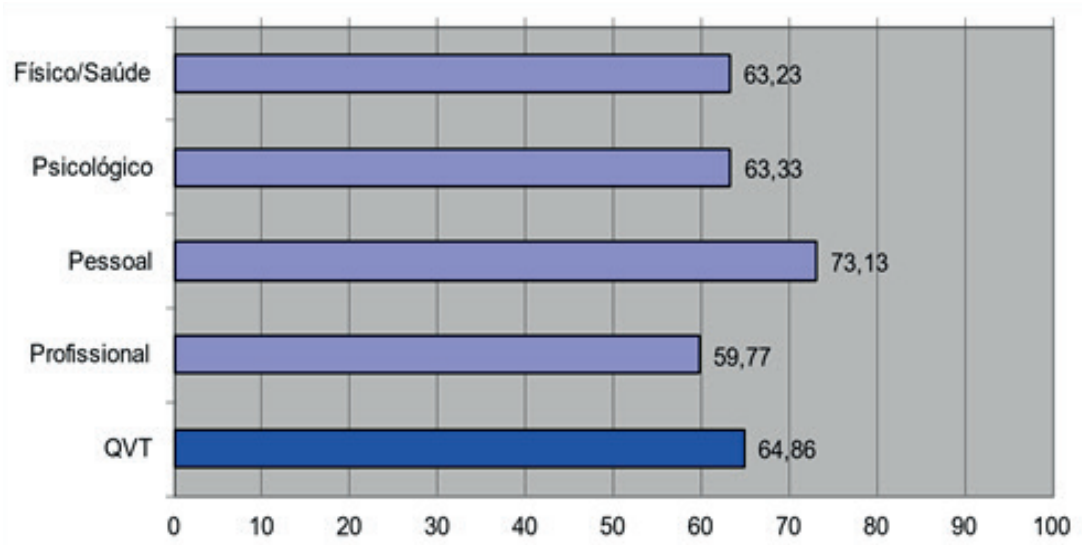

FIGURA 1: Escore dos domínios da avaliação da Qualidade de Vida no Trabalho na Unidade de Saúde da Família Integrando Vidas, em João Pessoa, Paraíba, 2021. Fonte: Pesquisa direta, João Pessoa, 2021.

A Tabela 3 evidencia a caracterização dos profissionais de saúde da ESF, identificando a distribuição de acordo com os dados sociodemográficos abordados e o escore de QVT total e de cada domínio avaliados pelo QWLQ-bref de acordo com cada perfil.

TABELA 3: Dados sociodemográficos e Escores da QVT totais e por cada Domínio de acordo com cada variável dos profissionais da USF Integrando Vidas, João Pessoa, Paraíba, em fevereiro de 2021.

\begin{tabular}{|c|c|c|c|c|c|c|}
\hline & $\begin{array}{c}\text { Profissionais } \\
\%(n)\end{array}$ & QVT & $\begin{array}{c}\text { Domínio } \\
\text { Físico/Saúde }\end{array}$ & $\begin{array}{c}\text { Domínio } \\
\text { Psicológico }\end{array}$ & $\begin{array}{c}\text { Domínio } \\
\text { Pessoal }\end{array}$ & $\begin{array}{c}\text { Domínio } \\
\text { Profissional }\end{array}$ \\
\hline Total & $100 \%(60)$ & 64,86 & 63,23 & 63,33 & 73,13 & 59,77 \\
\hline \multicolumn{7}{|l|}{ Sexo } \\
\hline Feminino & $71,7 \%(43)$ & 65,77 & 64,39 & 63,95 & 74,42 & 60,34 \\
\hline Masculino & $28,3 \%(17)$ & 62,48 & 61,98 & 61,98 & 69,53 & 59,03 \\
\hline \multicolumn{7}{|l|}{ Idade (anos) } \\
\hline $20-29$ & $25 \%(15)$ & 71,40 & 66,07 & 69,64 & 79,46 & 70,44 \\
\hline $30-39$ & $28,3 \%(23)$ & 59,64 & 58,52 & 57,58 & 68,18 & 54,29 \\
\hline $40-49$ & $40 \%(24)$ & 64,29 & 63,02 & 63,54 & 73,44 & 57,18 \\
\hline $50-59$ & $8,3 \%(5)$ & 63,61 & 61,25 & 66,67 & 68,75 & 57,78 \\
\hline$>60$ & $5 \%(3)$ & 66,78 & 75,00 & 58,33 & 70,83 & 62,96 \\
\hline \multicolumn{7}{|l|}{ Cor } \\
\hline Preta & $10 \%(6)$ & 67,42 & 61,46 & 72,22 & 73,96 & 62,04 \\
\hline Parda & $50 \%(30)$ & 62,81 & 64,58 & 60,00 & 70,63 & 56,02 \\
\hline Indígena & $1,7 \%(1)$ & 63,37 & 56,25 & 66,67 & 75,00 & 75,28 \\
\hline Branca & $28,3 \%(23)$ & 66,59 & 61,65 & 65,15 & 75,28 & 64,27 \\
\hline \multicolumn{7}{|l|}{ Estado civil } \\
\hline Solteira(o) & $35 \%(21)$ & 67,95 & 64,06 & 66,67 & 75,94 & 65,14 \\
\hline Casada(o) ou união estável & $51,7 \%(31)$ & 62,49 & 60,66 & 62,01 & 70,77 & 56,54 \\
\hline Separada(o) ou viúva(o) & $13,3 \%(8)$ & 71,92 & 75,89 & 67,86 & 77,68 & 66,27 \\
\hline
\end{tabular}


Continuação Tabela 3

\begin{tabular}{|c|c|c|c|c|c|c|}
\hline \multicolumn{7}{|l|}{ Escolaridade } \\
\hline Ensino médio & $50 \%(30)$ & 66,82 & 62,93 & 65,52 & 76,08 & 62,74 \\
\hline Ensino técnico & $5 \%(3)$ & 63,66 & 66,67 & 66,67 & 66,67 & 54,63 \\
\hline Ensino superior & $18,3 \%(11)$ & 62,97 & 64,42 & 59,62 & 69,71 & 58,12 \\
\hline Pós-graduação & $26,7 \%(16)$ & 66,05 & 64,38 & 66,26 & 74,69 & 58,89 \\
\hline \multicolumn{7}{|l|}{ Categoria profissional } \\
\hline ACS & $41,7 \%(25$ & 62,52 & 62,50 & 61,33 & 70,25 & 56,00 \\
\hline ASB & $3,3 \%(2)$ & 74,65 & 65,63 & 75,00 & 84,38 & 73,61 \\
\hline Auxiliar Adm. & $6,7 \%(4)$ & 79,64 & 75,00 & 81,25 & 85,94 & 76,39 \\
\hline Téc. enf./ lab. & $5 \%(3)$ & 47,11 & 52,08 & 47,22 & 52,08 & 37,04 \\
\hline Enfermeira(o) & $8,3 \%(5)$ & 55,56 & 57,50 & 48,33 & 62,50 & 53,89 \\
\hline Dentista & $5 \%(3)$ & 65,34 & 58,33 & 69,44 & 77,08 & 56,48 \\
\hline Médica(o) & $8,3 \%(5)$ & 65,97 & 71,25 & 58,33 & 78,75 & 55,56 \\
\hline Estudante & $10 \%(6)$ & 72,05 & 60,42 & 70,83 & 83,33 & 73,61 \\
\hline Equipe Multi. & $11,7 \%(7)$ & 68,61 & 64,58 & 69,44 & 76,04 & 64,35 \\
\hline \multicolumn{7}{|l|}{ Vínculo empregatício } \\
\hline Estatutário & $45 \%(27)$ & 61,65 & 62,26 & 60,58 & 68,51 & 55,24 \\
\hline Celetista & $33,3 \%(20)$ & 65,88 & 63,99 & 63,49 & 75,60 & 60,45 \\
\hline Bolsista & $11,7 \%(7)$ & 67,61 & 66,96 & 66,67 & 74,11 & 62,70 \\
\hline Estagiário & $10 \%(6)$ & 72,05 & 60,42 & 70,83 & 83,33 & 73,61 \\
\hline \multicolumn{7}{|c|}{ Tempo no serviço (anos) } \\
\hline 0 a 9 & $45 \%(27)$ & 68,76 & 66,20 & 66,67 & 75,55 & 64,61 \\
\hline 10 a 19 & $48,3 \%(29)$ & 61,49 & 60,99 & 59,48 & 69,83 & 55,65 \\
\hline$>20$ & $6,7 \%(4)$ & 63,06 & 59,38 & 68,75 & 67,19 & 56,94 \\
\hline \multicolumn{7}{|l|}{ Exerce outro trabalho } \\
\hline Sim & $35 \%(21)$ & 62,71 & 59,82 & 61,51 & 71,73 & 57,80 \\
\hline Não & $65 \%(39)$ & 66,02 & 65,06 & 64,32 & 73,88 & 60,83 \\
\hline \multicolumn{7}{|l|}{ Carga horária semanal } \\
\hline $20-40$ & $80 \%(48)$ & 64,09 & 63,39 & 61,73 & 71,68 & 59,57 \\
\hline$>40$ & $20 \%(12)$ & 68,11 & 61,98 & 70,14 & 79,69 & 60,65 \\
\hline \multicolumn{7}{|c|}{ Renda fam. (salário mín.) } \\
\hline até 1 & $5 \%(3)$ & 57,58 & 56,25 & 52,78 & 66,67 & 54,63 \\
\hline 1 a 3 & $43,3 \%(26)$ & 69,35 & 65,87 & 71,15 & 75,96 & 64,42 \\
\hline 3 a 6 & $21,7 \%(13)$ & 61,76 & 60,71 & 55,95 & 71,43 & 58,93 \\
\hline 6 a 9 & $10 \%(6)$ & 65,65 & 67,71 & 63,89 & 70,83 & 60,19 \\
\hline 9 a 12 & $8,3 \%(5)$ & 56,63 & 60,00 & 51,67 & 63,75 & 51,11 \\
\hline$>12$ & $10 \%(6)$ & 62,41 & 57,29 & 63,89 & 77,08 & 51,39 \\
\hline não informou & $1,7 \%(1)$ & 69,79 & 81,25 & 50,00 & 81,25 & 66,67 \\
\hline \multicolumn{7}{|l|}{ Pessoas em casa } \\
\hline 1 a 2 & $25 \%(15)$ & 62,72 & 61,25 & 57,78 & 70,00 & 61,85 \\
\hline 3 a 4 & $61,7 \%(37)$ & 65,50 & 63,34 & 65,54 & 74,49 & 58,63 \\
\hline$>5$ & $13,3 \%(8)$ & 65,93 & 66,41 & 63,54 & 72,66 & 61,11 \\
\hline \multicolumn{7}{|l|}{ Residência (bairro) } \\
\hline João Paulo II & $18,3 \%(11)$ & 61,27 & 61,93 & 59,85 & 66,48 & 56,82 \\
\hline Geisel & $15 \%(9)$ & 59,99 & 61,81 & 57,41 & 67,36 & 53,40 \\
\hline Funcionários II & $20 \%(12)$ & 64,38 & 61,46 & 62,50 & 75,00 & 58,56 \\
\hline Outro & $46,7 \%(28)$ & 68,05 & 64,96 & 66,96 & 76,79 & 63,49 \\
\hline \multicolumn{7}{|c|}{ Presença de comorbidade } \\
\hline Sim & $28,3 \%(17)$ & 61,38 & 57,35 & 61,27 & 69,85 & 57,03 \\
\hline Não & $71,7 \%(43)$ & 66,24 & 65,55 & 64,15 & 74,42 & 60,85 \\
\hline
\end{tabular}


Dos 60 trabalhadores, a maioria são mulheres (71,7\%), com índice QVT considerado satisfatório. Sobre a idade, 15 (25\%) trabalhadores possuem de 20 a 29 anos, com escore QVT de 71,40, mais de cinco pontos maior que a média total, o maior entre as faixas etárias, principalmente pelos domínios psicológico e pessoal; 13 (21,7\%) de 30 a 39 anos, com o menor escore, 59,64, mais de cinco pontos a menos de que a média total, principalmente pelo domínio profissional, que se avaliado isoladamente é considerado neutro, uma categoria inferior à QVT total.

A satisfação com o trabalho se relaciona também a aspectos da vida pessoal do tra-balhador, com interferência, ainda, da situação social e demográfica. ${ }^{2}$ Dentre as variáveis sociodemográficas do estudo que sugere uma relação positiva com a QVT, podese inicialmente notar a percepção de QVT mais satisfatória entre a faixa etária de 20 a 29 anos. Já o grupo etário compreendido entre os 30 e 39 anos apresenta, em seu domínio profissional, classificação neutra, seguindo os demais grupos com classificação semelhante à da média.

Em relação ao estado civil, 21 (35\%) são solteiros, 31 (51,7\%) casados ou em união estável e oito (13,3\%) viúvos ou separados, tendo essa última categoria índice de QVT de 71,92 , com aumentos significativos nos índices principalmente dos domínios físico/saúde e profissionais, em relação à média total. No quesito escolaridade, 30 (50\%) possuem ensino médio, três (5\%) ensino técnico, 11 $(18,3 \%)$ ensino superior e $16(26,7 \%)$ pósgraduação, todos sem variação importante entre as médias dos domínios, mas com os de ensino técnico com um índice inferior no domínio profissional, de 54,63, considerado neutro.

Em relação à escolaridade, é reiterado pela literatura o relato de profissionais com menores graus acadêmicos apresentarem índices de QVT mais baixos. ${ }^{2}$ Apesar disso, neste estudo foi observado que o grau médio apresenta nível de satisfação maior do que outros graus de instrução no domínio profissional, mesmo com escolaridade menor, diferindo do esperado neste quesito.

Entre as categorias profissionais, 25 (41,7\%) dos entrevistados atuam como Agentes Comunitários de Saúde (ACS), dois (3,3\%) como Auxiliares de Saúde Bucal (ASB), quatro (6,7\%) como auxiliares administrativos, três (5\%) atuam como técnicos de enfermagem ou de laboratório, cinco $(8,3 \%)$ como enfermeiros, três (5\%) como cirurgiões-dentistas, cinco $(8,3 \%)$ como médicos, sete $(11,7 \%)$ como trabalhadores de equipe multidisciplinar (duas nutricionistas, um fisioterapeuta, duas psicólogas, duas farmacêuticas) e seis (10\%) estudantes do curso de graduação em medicina, em estágio obrigatório.

A principal composição desse grupo etário na unidade em questão é de estudantes de graduação em medicina, residentes médicos e multiprofissionais e profissionais há pouco tempo formados. Isso resulta no contato menos duradouro com os problemas a serem diariamente enfrentados no âmbito da ESF, sem sobrecarga acumulada, sem vínculo empregatício direto com a gestão, por serem majoritariamente bolsistas ou estagiários, e com perspectiva de um ofício passageiro na unidade, pois não necessariamente permanecerão na unidade quando finalizado o curso, fatores que podem propiciar uma menor pressão frente às tensões e fatores que podem afetar a percepção da QVT.

As diferenças significativas de escore de QVT são encontradas na categoria de auxiliares administrativos, com índice de 79,64, classificado como muito satisfatório, um nível acima que a média total, principalmente devido ao domínio pessoal e psicológico, e na categoria de técnicos de 
enfermagem e laboratório, com QVT de 47,11, classificada como neutro, um nível inferior em relação à média total, principalmente devido ao domínio profissional, este isoladamente com índice de 37,04 nesta categoria, classificado como insatisfatório.

O grupo que demonstra percepção mais positiva da QVT é o de auxiliares administrativos, única variável que foi classificada como muito satisfatória nos domínios pessoal, psicológico e na média de todos. A saúde psicológica é repleta de subjetividade e abrange todos os âmbitos em que o indivíduo se insere, e sua manutenção se constitui em valiosa ponte entre o bemestar nas esferas pessoais e profissionais, com importante influência da preservação de boas relações e de boa comunicação no trabalho. ${ }^{10} \mathrm{~A}$ percepção de suas esferas pessoais e psicológicas como muito satisfatórias no trabalho eleva a qualidade de vida dos auxiliares administrativos, podendo ser destacado também que suas atividades predominantemente envolvem atos administrativos, como recebimento de solicitações de exames, recepção e orientação aos usuários e dispensação de medicamentos, com menor nível de tensão perante o usuário.

A categoria dos técnicos de enfermagem, ou de laboratório foi a única que obteve classificação insatisfatória em um de seus domínios, o profissional, acompanhada da categoria da enfermagem, com classificação neutra neste domínio, inferior às demais profissões, todas com avaliação satisfatória. A falta de condições ideais para o exercício laboral, com alta demanda por resultados, o desenfreado ritmo de trabalho e os eventuais deslizes nas relações socioprofissionais somado à insuficiente autonomia dos trabalhadores de enfermagem na ESF, podem influenciar na eficiência e segurança da assistência e sua
QVT7.

Os trabalhadores que afirmam seu vínculo empregatício ser estatutário são 27 (45\%), celetista são 20 (33,3\%), bolsista são sete $(11,7 \%)$ e estagiário são seis (10\%), os quais possuem maior índice QVT que os demais, 72,05, mas ainda dentro da mesma classificação. 27 (45\%) possuem de zero a nove anos no serviço, 29 possuem de 10 a 19 anos, e quatro possuem mais de 20 anos. Os que exercem outro trabalho são 21 (35\%), e 39 (65\%) não exercem. A carga horária de $48(80 \%)$ é de até 40 horas semanais, e a de 12 (20\%) é maior que 40 horas semanais, mas sem implicância com os índices de QVT.

Ponto importante a ser lembrado é a fragilidade muitas vezes existente no vínculo empregatício não estatutário, eventualmente atrelado a questões políticas de interesses variados como sendo orientadora da organização laboral $^{4}$, âmbito que se relaciona ainda com má remuneração e impacta a satisfação desta e de outras classes profissionais, tão importantes no processo de trabalho da ESF. Lorenz ${ }^{14}$ considera a qualidade do trabalho da enfermagem como boa, mas reconhece a inadequação de recursos materiais e humanos, citando a síndrome do esgotamento profissional como preditora de menor qualidade do cuidado, da intenção de sair do atual emprego e de abandonar a enfermagem como profissão. ${ }^{15}$

A renda domiciliar de até um salário mínimo é a de três (5\%) participantes, refletindo um índice geral mais baixo dentro da mesma classificação satisfatória, de 57,58, mas um índice de domínio profissional de classificação inferior, de 54,63; 26 (43,8\%) possuem renda de um a três salários mínimos; $13(21,7 \%)$ possuem de três a seis salários mínimos; seis $(10 \%)$ possuem de seis a nove; cinco $(8,3 \%)$ possuem de nove a 12 e seis (10\%) possuem mais de 12 salários mínimos em renda domiciliar, com esses dois últimos 
grupos apresentando um índice de domínio profissional também de classificação inferior, com 51,11 e 51,39, respectivamente, mas com índice geral de QVT dentro da classificação satisfatória. Número de pessoas no domicílio é de uma a duas em 15 (25\%) participantes, de três a quatro em $37(61,7 \%)$ e de cinco ou mais em oito $(13,3 \%)$.

Os trabalhadores que não moram nos bairros próximos à unidade são 28 (46,7\%); dos que moram, $11(18,3 \%)$ residem no bairro João Paulo II, nove (15\%) no bairro Ernesto Geisel, e 12 no bairro Funcionários II, com os que moram no Geisel apresentando um escore no domínio profissional de 53,40, classificação inferior à média total. Dos participantes, 43 $(71,7 \%)$ não apresentam comorbidades, e 17 (28,3\%) apresentam, com as mais frequentes sendo hipertensão, diabetes, asma, depressão e ansiedade, mas sem correlação com variação de índice de QVT.

Muitos são os desafios e os fatores com potencial de dano e prejuízo à QVT na $E S F$, alguns deles elencados por Bacurau ${ }^{12}$, como a situação de risco e vulnerabilidade social e os conflitos sociais da comunidade assistida; metas a serem cumpridas, muitas vezes de caráter obrigatório para parte da remuneração, não sempre com insumos e estrutura essencial para seu cumprimento, e não sempre vinculada com a demanda prioritária da comunidade; condições de trabalho inadequadas e eventualmente não higiênicas e possíveis causadoras de adoecimento; dificuldades entre as redes de atenção e de capacitação continuada; e falta de escuta e de acolhimento ao empregado. ${ }^{12}$ Outros problemas são a sobrecarga de trabalho e a falta de equipamentos de proteção individuais (EPIs) adequados e de ideais condições de infraestrutura e de redes de apoio.

Aqualidadedasaúdedostrabalhadores e dos serviços e ações prestados aos usuários do sistema se torna, assim, dependente da maneira como os profissionais que nele atuam são tratados. ${ }^{10}$ No entanto, ao passo que a qualidade das ações na ESF e a correção de todos esses fatores descritos depende de políticas de incentivo, devendo, também entender a determinação do processo de trabalho concretizado nas redes de serviços e, consequentemente, a satisfação do trabalhador com seu serviço, como resultado da incorporação desses quesitos. ${ }^{15}$

No enfrentamento à situação de saúde pública vivida com a COVID-19, o fornecimento de EPIs, de treinamentos específicos para atuação em pandemia e de adequada especialização em saúde da família para todas as categorias da equipe multiprofissional, pela atuação complexa da saúde na ESF, são condições estritas para que se atinja ao máximo na eficácia da diminuição do risco de infecção e transmissão tanto entre os componentes da equipe como para a população, e sem tais condições sendo atendidas, o funcionamento da USF pode apresentar mais risco do que benefício nesta pandemia. ${ }^{11}$ Isso se configura em um motivo a mais que implica dificuldades no alcance de melhor QVT na ESF.

\section{CONCLUSÃO}

A Qualidade de Vida no Trabalho dos trabalhadores de saúde da USF Integrando Vidas foi considerada satisfatória, com o domínio pessoal com maior escore e o profissional com o menor. Esse resultado positivo pode estar relacionado as condições de trabalho na USF, que apresenta equipe de Saúde da Família completas, com programas 
de Residência Médica e Multiprofissional atuantes, com atividades de planejamento das ações, reuniões de equipe, atividades de promoção e prevenção, dentre outros. Ademais, os resultados apresentados nos domínios de qualidade de vida dos trabalhadores, principalmente relacionado

\section{REFERÊNCIAS BIBLIOGRÁFICAS}

1. Ferro FF, Duarte FM. Instrumentos para medir a qualidade de vida no trabalho e a ESF: uma revisão de literatura. Universidade Federal de Minas Gerais. Faculdade de Medicina. Núcleo de Educação em Saúde Coletiva. Brumadinho, 2012. 92f. Monografia (Especialização em Atenção Básica em Saúde da Família). Disponível em: https://www.nescon. medicina.ufmg.br/biblioteca/imagem/3756.pdf.

2. Barbosa ML, MENEZES TN, Santos SR, Olinda RA, Costa GMC. Qualidade de vida no trabalho dos profissionais de saúde no sistema prisional. Ciênc saúde coletiva. 2018, 23(4): 1293-02. Disponível em: https://www.scielosp.org/pdf/csc/2018.v23n4/12931302.

3. Cheremeta M, Pedroso B, Pilatti LA, Kovaleski JL. Construção da versão abreviada do QWLQ-78: um instrumento de avaliação da qualidade de vida no trabalho. R bras Qual Vida. 2011, 3(1): 01-15. Disponível em: https://periodicos.utfpr.edu.br/rbqv/article/ view/758.

4. Lacaz FAC, Vieira NP, Cortizo CT, Junqueira V, Santos APL, Santos FS. Qualidade de vida, gestão do trabalho e plano de carreira como tecnologista em saúde na atenção básica do Sistema Único de Saúde em São Paulo, Brasil. Cad Saúde Pública. 2010, 26(2): 253-63. Disponível em: https://www. scielo.br/scielo.php?script=sci_arttext\&pid=S0102311X2010000200005.

5. Reis Júnior DR. Qualidade de vida no trabalho: construção e validação do questionário QWLQ78. Universidade Tecnológica Federal do Paraná. ao pessoal, podem estar relacionados a faixa etária, estado civil, remuneração, categoria profissional e vínculo empregatício. Nesse sentido, os trabalhadores mais jovens, viúvos/separados ou solteiros, estagiários e bolsistas apresentaram scores maiores na Qualidade de Vida no Trabalho.

Programa de Pós-Graduação em Engenharia de Produção. Ponta Grossa, 2008. 114f. Dissertação (Mestrado em Engenharia de Produção). Disponível em: http://www.pg.utfpr.edu.br/ppgep/dissertacoes/ arquivos/101/Dissertacao.pdf.

6. Teles MAB, Barbosa MR, Vargas, AMD, Gomes VE, Ferreira EF, Martins AMEB, Ferreira RC. Psychosocial work conditions and quality of life among primary health care employees: a cross sectional study. Health Qual. Life Outcomes. 2014, 12: 72. Disponível em: https://doi.org/10.1186/1477-7525-12-72.

7. Marques ALN, Ferreira MBG, Duarte JMG, Costa NS, Haas VJ, Simões ALA. Qualidade de vida e contexto de trabalho de profissionais de enfermagem da Estratégia Saúde da Família. Rev Rene. 2015, 16(5): 672-81. Disponível em: https://scielosp.org/article/ sdeb/2019.v43n122/765-779/.

8. Peduzzi M. Trabalho e educação na saúde: ampliação da abordagem de recursos humanos. Ciênc saúde coletiva. 2013, 18(6): 1535-1543. Disponível em: https://doi.org/10.1590/S1413-81232013000600005.

9. Pires DEPP, Machado RR, Soratto J, Schere MA, Gonçalves ASF, Trindade LL. Cargas de trabalho da enfermagem na saúde da família: implicações no acesso universal. Rev Latino-Am Enfermagem. 2016, 24: e2682. Disponível em: https://www.scielo.br/pdf/ rlae/v24/pt_0104-1169-rlae-0992-2682.pdf.

10. Gomes MFP, Mendes ES, Fracolli LA. Qualidade de vida dos profissionais que trabalham na Estratégia Saúde da Família. Rev Aten Saúde. 
12016, 14(49): 27-33. Disponível em: https://www. scielo.br/pdf/reben/v73n5/pt_0034-7167-reben-7305-e20190645.pdf.

11. Soares CSA, da Fonseca CLR. Atenção primária à saúde em tempos de pandemia. J Manag Prim Health Care. 2020, 12: 1-11. Disponível em: https:// www.jmphc.com.br/jmphc/article/view/998.

12. Bacurau FRS, Melo Neto AJ, Ahuiar FB, HirschMonteiro C. Qualidade de vida de trabalhadores de Unidades de Saúde da Família. Saúde Meio Ambient. 2017, 5(2): 127-40. Disponível em: https://periodicos. ufms.br/index.php/sameamb/article/view/5043.

13. Bracarense CF, Costa NS, Duarte JMG, Ferreira
MBG, Simões ALS. Qualidade de vida no trabalho: discurso dos profissionais da Estratégia Saúde da Família. Esc Anna Nery. 2015, 19(4):542-48. Disponível em: https://doi.org/10.5935/1414-8145.20150072.

14. Lorenz VR, Sabino MO, Corrêa Filho HR. Esgotamento profissional, qualidade e intenções entre enfermeiros de saúde da família. Rev Bras Enferm. 2018, 71(5): 2295-01. Disponível em: https:// doi.org/10.1590/0034-7167-2016-0510Carrapato JFL.

15. Castanheira ERL, Placideli N. Percepções dos profissionais de saúde da atenção primária sobre qualidade no processo de trabalho. Saude soc. 2018, 27(2): 518-30. Disponível em: https://doi.org/10.1590/ s0104-12902018170012. 